\title{
Combatting the Pathological Brain Microbiome
}

\section{Shimon Shatzmiller*}

Department of Chemical Sciences, Ariel University, Ariel, Israel

*Corresponding Author: Shimon Shatzmiller, Department of Chemical Sciences, Ariel University, Ariel, Israel.

Received: May 21, 2019; Published: July 09, 2019

DOI: $10.31080 /$ ASNE.2019.02.0078

\begin{abstract}
Substances that come from nutrients, to combat inflammation and the crawling infection might be a proper approach to treat The quest of remedy for neurodegenerative diseases is already more than a century old. It is unclear, applying the moist sophisticated ultramodern methods and equipment, what is in fact going on in the human brain when this curse hits. However, Nowadays the perception is that gut microbiome penetrates the brain via the "Gut-Brain" axis. Either by using nerves or through the bloodstream by moving from a leaky gut to a damaged blood-brain barrier. Moreover, when in the brain, causing inflammation and subsequent infection, that moves in the brain in a prion-like a mechanism, from one cell to its neighbor until interoception (death) is reached.

The eradication of bacteria antimicrobial peptide surrogates in synergy with antioxidants, neurodegeneration at its early stages.

Like other parts of our body, the brain is home to bacteria, viruses and fungus, such as yeast and larger parasites such as amoebas. This changes the clinical landscape of many conditions, including autism, neurodegenerative disease such as Alzheimer's and Parkinson's and chronic inflammatory response syndromes (CIRS). It also forces us to consider the 'mind', 'self', and 'agency' blurring the lines between science and philosophy.
\end{abstract}

Keywords: Microbiome; Organisms
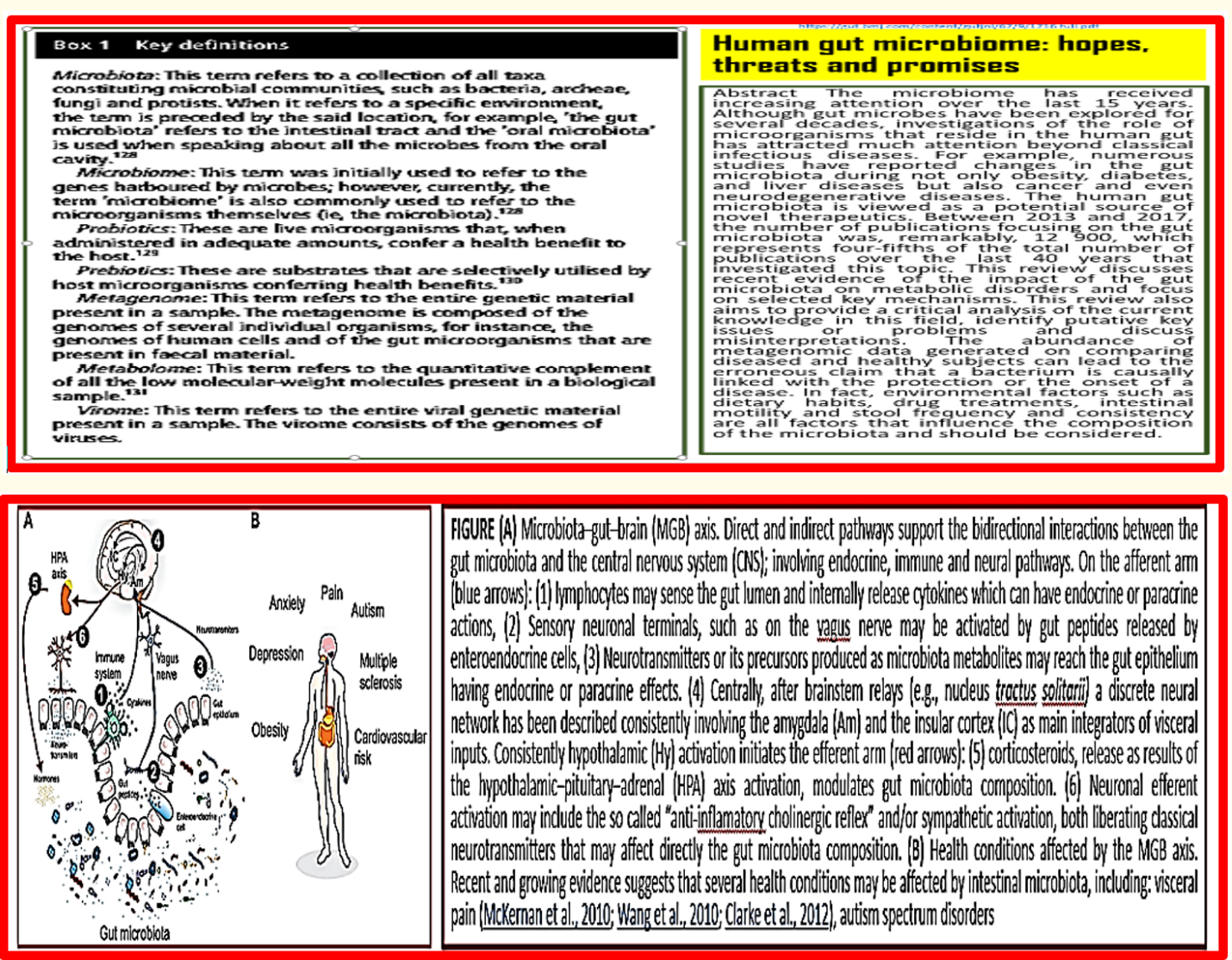

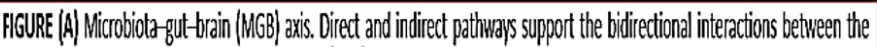
gut micobobiota and the central nervous ssstem (ars); involing endocine, immune and neural pathwass. On the afferent amm

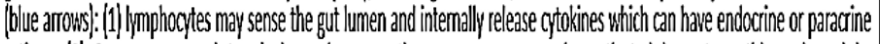
actions, (2) Sensory neuronal terminals, such as on the vagas nevere may be activated by gut peptides released by enteroendocine cells, (3) Neurotransmitters or its precussors produced as microbiota metabolites may reach the gut epithelium having endocrine or paracine effects. (4) Centrally, atter brinstem relas (e.g, nucleus troctus solitarii) a discrele neural network has been described consistently involing the amygdala (Am) and the insular cortex (C) as main intergators of visceral inputs. Consitenty hypothalamic (Hy) activation intiates the efferent arm |red arrouss): (5) coticosteroids, release as results of the hypothalamic-pitutitan-adrenal (HPA) axis attiration, modulates gut microtiota composition. (6) Neuronal efferent activation may indude the so called "anti-inflamatory cholinergic ceflex" and/or sympathetic attivation, both liberting dassical neurotransmitters that may affect directly the gut microbiota composition. (B) Heath conditions affected by the MGB axis.

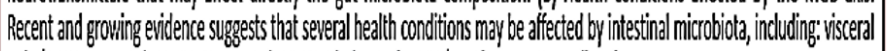
pain (Mckernan etal, 2010; Wangetal, 2010: Clarketetal, 2012), autism spectrum disorders

Figure 1 
Microbiome system offers information to learn more about the microscopic organisms and their behavior in your gut. Think of it as the story of our inner world-a snapshot of what's happening inside the ower body. New research about the microbiome is changing the understanding of who you are as a human and how your body functions" (credit ref. [1]).

From Crohn's disease to asthma, obesity or even type 2 diabetes, gut microbiota dysbiosis has already been linked to many human diseases. Even to mental health and behavior. Previous research studies have already shown that there is a connection between our gut and our brain. However, as they have been done using animal models, could these results found in mice be equally demonstrated in humans? Are they to extrapolate?

Moreover, that is precisely what researchers of the Catholic University of Leuven, in Belgium, have just done. They have found most human gut bacteria do produce neurotransmitters, which are chemicals like dopamine and serotonin that enable communication among neurons, which are the nervous cells in the brain, but also in the enteric nervous system of the gut. These neurotransmitters are known to influence intestinal functions, but also our mood and behavior"(credit ref. [2-4]).

Recent research showed that there are gut microbiome alterations in Alzheimer's disease [5].

A limited number of studies have attempted to address the role of gut microbiota in AD. A recent investigation in cognitivelyimpaired older adults (without an AD diagnosis) reported an increased abundance of the pro-inflammatory bacteria Escherichia/
Shigella and decreased the abundance of the anti-inflammatory bacteria EU bacterium recall in individuals with evidence of amyloid deposition on PET imaging compared to individuals [6]: who were amyloid negative [7]. However, while the anatomy and physiology of the gastrointestinal tract of humans and mice share many characteristics, there are also substantial differences concerning resident bacterial communities 30 , which makes comparing taxa and changes in abundance between these studies difficult" (credit ref. [3]).

It seems that microbiome research, in particular Gut microbiome, is gaining momentum in the direction of early detection and followup for therapy in the neurodegeneration area, based on the finding that gut bacterias of different lines are occupying both gut and brainpan and the composition in the diseases progress respectively.

Foreword: Our brain is home for microbiome dwellers

The brain microbiome is the array of microbes that live in symbiosis with our neurons and glial cells. This is an entirely novel concept that had never been described until the publication in 2016 [8] and has been dubbed "the fourth brain".

The brain-gut axis reflects the bidirectional, constant communication between the central nervous system (CNS) and the gastrointestinal tract. There is also growing evidence that the intestinal microbiota influences the brain-gut interactions in different points of time (from early life to neurodegeneration), as well as at different levels (from the gut lumen to the CNS) [9].

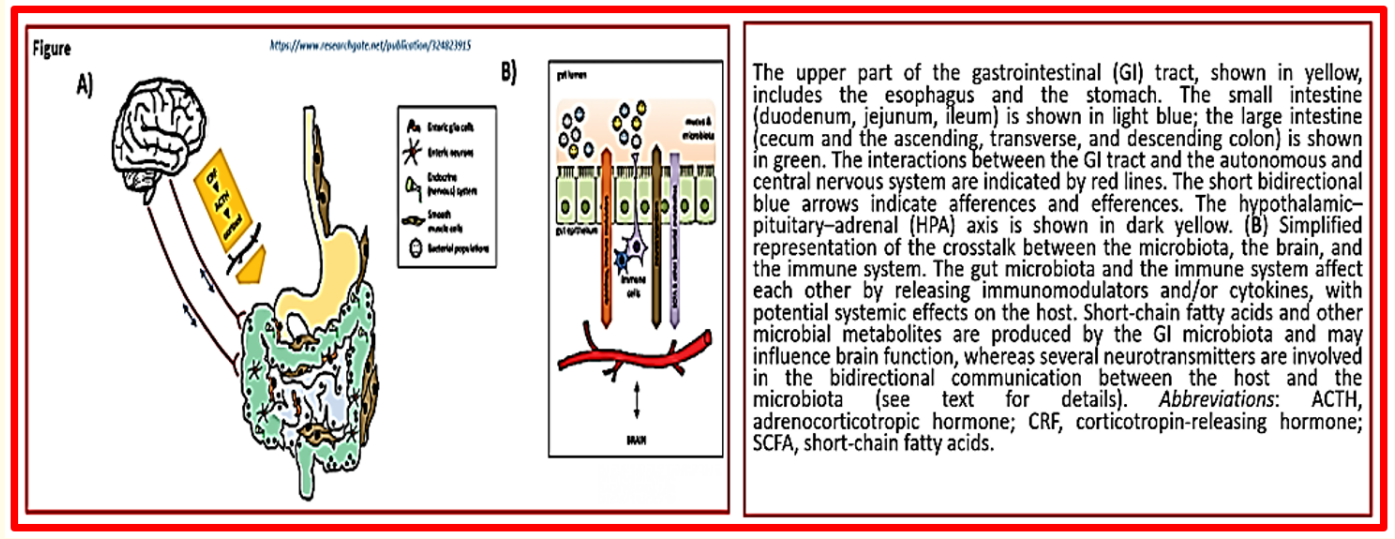

Figure 2 
Harvard researchers, Dr. Rudolph Tanzi and Robert D. Moir, $\mathrm{PhD}$, are heading up a team, funded by the Cure Alzheimer's Fund and the Good Ventures Foundation, that has taken on mapping the microbiome, the population of microorganisms, some helpful and some pathological, that exists inside the brain [10].

Increasingly, we recognize the importance of the microbiome - the bacterial (and other) flora that lives within us, sometimes in conflict, often in symbiosis, always interacting. Whether the microbiome changes drive changes in our health, or vice versa, is not often known. However, figuring out what a healthy microbiome looks like or how it can evolve in the time of disease can help us better understand the minutiae of these interactions.

The human microbiome (Gut microbiota) influences our brain health in numerous ways: [11]
1. Substances of microbes, like lipopolysaccharides, provide low-grade tonic stimulation of the innate immune system.

2. Bacterial proteins and peptides.

3. Bacterial enzymes.

4. Gut microbes can produce agents like hormones and neurotransmitters

5. Gut bacteria directly stimulate afferent neurons of the enteric

Antimicrobial peptides (AMPs) are antibiotics that eradicate microorganisms. The short, cationic peptides have been implicated in many biological processes, primarily involving innate immunity.

Recent investigations identified bacteria from different lines in the inner brain. The following table shows some examples. By sheer numbers, human bodies are awash in bacteria [12].

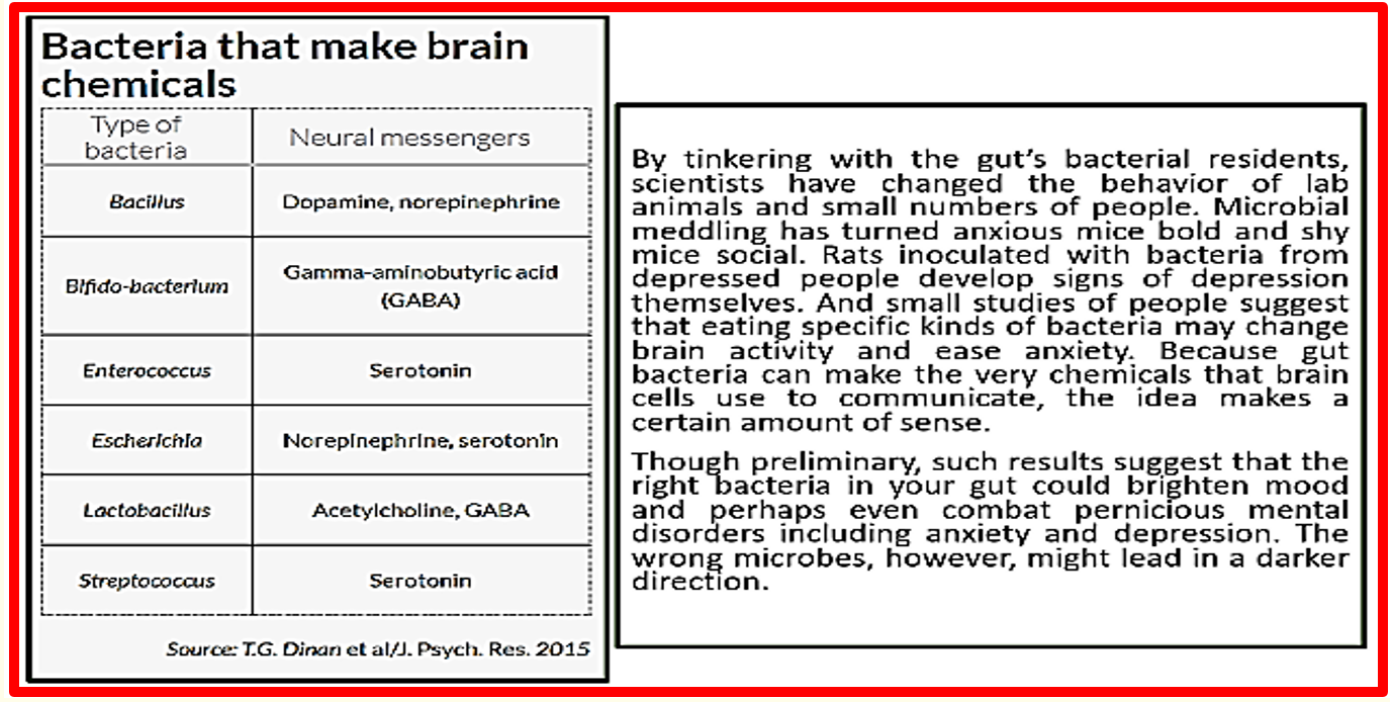

Figure 3

The penetration of microbes into the inner brain can be the onset of inflammation followed by infection in the brain. One can rationalize the digestion of APP and other proteins by enzymes to form antimicrobial peptides like the amyloid $b$ which combat the microbes, but aggregate to form many sorts of stable formations that damage the synapses as depicted above. The series of events that are induced by the oxidative stress of the inflammation, lead to the harming antimicrobial peptides of which the amyloids are only one sort. Since peptides can barely enter into the brain via the BBB [13]. However, it is possible to enter the brain via various receptors, an example is the diazepine receptor [14].

The physiological task of inflammation is to eliminate the initial cause of cell injury, clear out necrotic cells and tissues damaged from the original insult and the inflammatory process, and initiate tissue repair. 
In innate immunity, inflammation-based immunity is the first line of vertebrate defense against micro-organisms. Inflammation relies on several cellulars and molecular effectors that can strike invading pathogens very shortly after the encounter between inflammatory cells and the intruder, but in a non-specific way. inflammation can generate substantial costs for the host if the inflammatory response, and the associated oxygen-based damage, get out of control. This imposes strong selection pressure that acts to optimize two key features of the inflammatory response: the timing of activation and resolution (the process of downregulation of the response). Nevertheless, host immune regulation also opens the way to pathogens to subvert host defenses.
Currently, many agree that the gut microbiome reaches the brain using the "gut-brain axis" and is the cause for the onset of neurodegeneration - this penetration results in inflammation, then infection and apoptosis of the brain tissue. Antimicrobial peptide surrogates $[15,16]$, based on a brain-penetrating scaffold-like diazepine $[17,18]$ or 1,4 dihydropyridine [19-21] may be suitable to combat the invading microbiota and in a later stage serve as antimicrobial agents to combat the infection and prevent aggregation [22]. It was shown that some selectivity in bacteria eradication was possible [23].

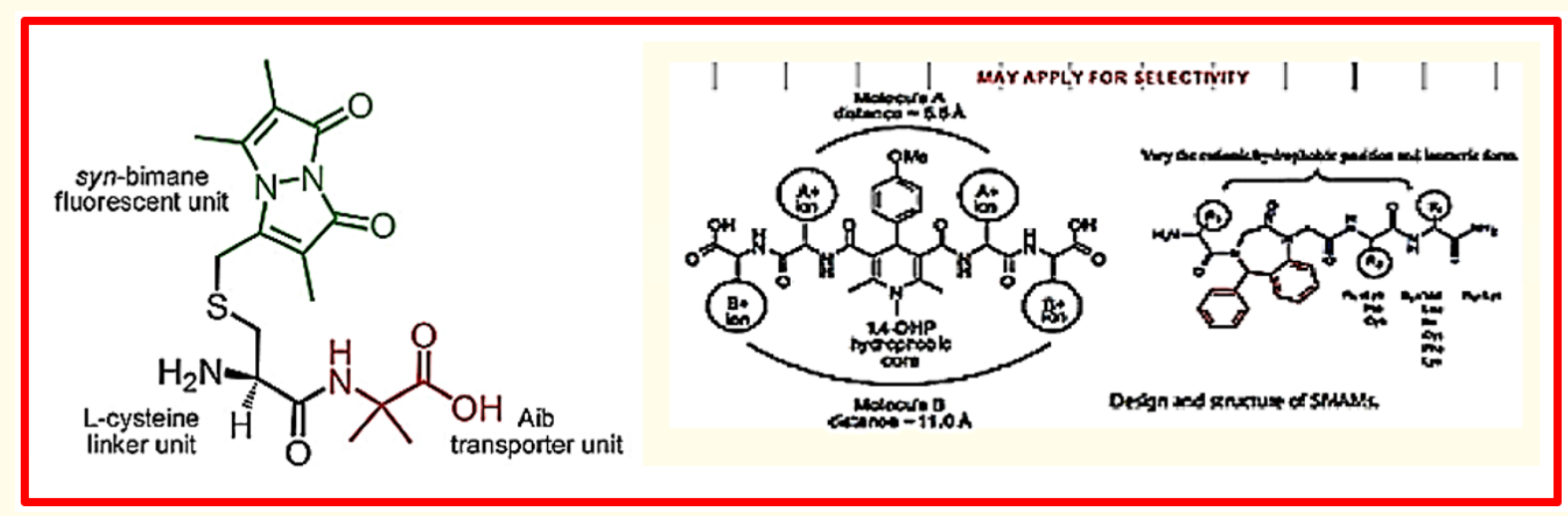

Figure 4: Peptide surrogates that cross BBB (credit ref. $[15,17,20])$.

Interdomain communication [24]

How have bacteria facilitated the origin and evolution of animals; how do animals and bacteria affect each other's genomes; how does normal animal development depend on bacterial partners; how is homeostasis maintained between animals and their symbionts; and how can ecological approaches deepen our un- derstanding of the multiple levels of animal-bacterial interaction. As answers to these fundamental questions emerge, all biologists will be challenged to broaden their appreciation of these interactions and to include investigations of the relationships between and among bacteria and their animal partners as we seek a better understanding of the natural world.

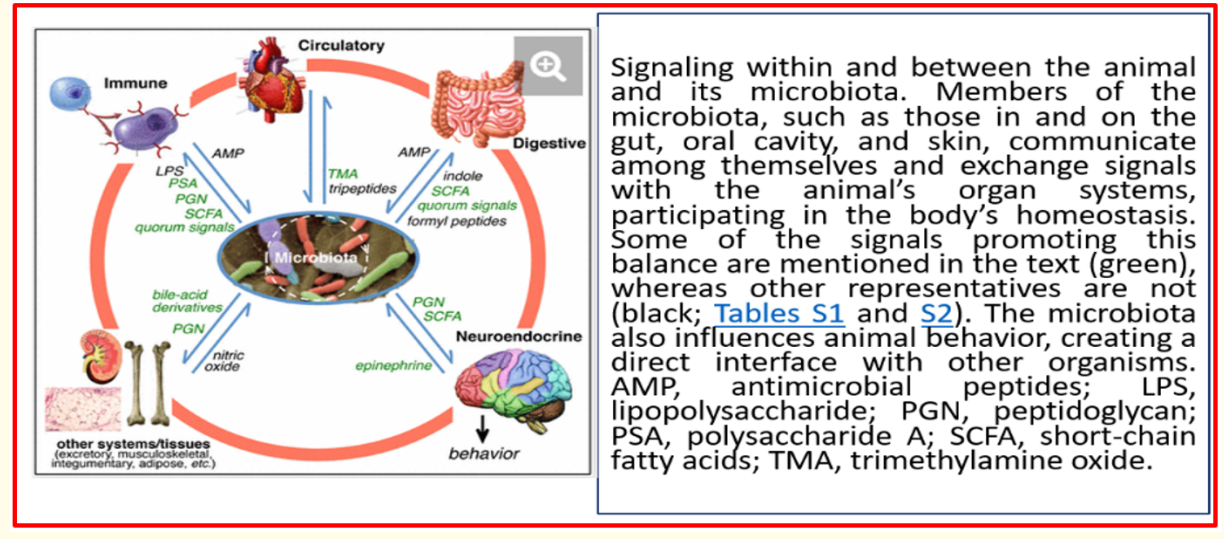

Figure 5 
Could some of these same bacteria settle in our brains?

A poster presented at the annual meeting of the Society for Neuroscience drew attention with high-resolution microscope images of bacteria penetrating and inhabiting the cells of healthy human brains.

The presented work is preliminary, and its authors are careful to note that their tissue samples, collected from cadavers, could have been contaminated [25].

The role of nutrition in the microbiota's gut-brain axis [26].

The microbial population dwelling in the small and large intestine represents the largest microbial population of the human microbiota. Moreover, the genes encoded by the gut micro-biota, the gut microbiome, outnumber the human genome by one hundred to one.

This complex eco-system is formed mainly by bacteria, but also viruses, archaea, protozoa and fungi.

Due to the advances in genomic technologies it has been possible to unravel around $75 \%$ of the adult gut microbiota bacterial composition, which is predominantly composed of the Firmicutes and Bacteroidetes phyla [27]).

Fecal microbiota transplantation (FMT) is the infusion of liquid filtrate feces from a healthy donor into the gut of a recipient to cure a specific disease [28-30].

\section{Conclusion}

In spite of the generally accepted dogma that the brain is a sterile environment, more data accumulates that proves our brain to be a dwelling place for bacteria, in particular gut microbes. Those may be the omns3et for many neurodegenerative diseases. Hinting that antimicrobial therapy might be applicable to many of the as for today "uncurable" diseases.

\section{Bibliography}

1. "10 Things You Can Learn From Your Microbiome".

2. https://www.gutmicrobiotaforhealth.com/en/scientistsidentified-two-bacteria-from-gut-microbiota-linked-to-mental-health/

3. Mireia Valles-Colomer., et al. "The neuroactive potential of the human gut microbiota in quality of life and depression". Nature Microbiology 4.4 (2019): 623-632.
4. Shimon E Shatzmiller. "Gut Microbes Start Neurodegeneration - The Inflammation Approach". EC Pharmacology and Toxicology SI.01 (2017): 01-03.

5. Nicholas M Vogt., et al. "Gut microbiome alterations in Alzheimer's disease". Scientific Reports volume 7.1 (2017): 13537.

6. Yuhai Zhao and Walter J Lukiw. "Bacteroidetes Neurotoxins and Inflammatory Neurodegeneration". Molecular Neurobiology 55.12 (2018): 9100-9107.

7. Cattaneo A., et al. "Association of brain amyloidosis with proinflammatory gut bacterial taxa and peripheral inflammation markers in cognitively impaired elderly". Neurobiology of Aging 49 (2017): 60-68.

8. Marco Ruggiero." Fecal Microbiota Transplantation and the Brain Microbiota in Neurological Diseases". Clinical Endoscopy 49.6 (2016): 579.

9. Karol Kowalski and Agata Mulak. "Brain-Gut-Microbiota Axis in Alzheimer's Disease". Journal of Neurogastroenterology and Motility 25.1 (2019): 48-60.

10. “Mapping The Brain's Microbiome: Can Studying Germs In The Brain Lead To A Cure For Alzheimer's?".

11. Leo Galland. "The Gut Microbiome and the Brain". Journal of Medicinal Food 17.12 (2014): 1261-1272.

12. Science News. 189.7 (2016): 23,

13. William A Banks. "Peptides and the blood-brain barrier". Peptides 72 (2015): 16-19.

14. Moshe Gavish., et al. "Enigma of the Peripheral Benzodiazepine Receptor". Pharmacological Reviews 51.4 (1999): 629650.

15. Shimon Shatzmiller., et al. "Combatting the Microbial Onset of Neurodegeneration the Peptide Surrogate Approach". EC Pharmacology and Toxicology 6.3 (2018): 152-184.

16. Shimon Shatzmiller*., et al. "Antibacterial Peptide Surrogates A Brief Review". EC Pharmacology and Toxicology 4.3 (2017): 94-111.

17. GalinaM Zats., et al. "Antimicrobial benzodiazepine-based short cationic peptidomimetics". Journal of Peptide Science 21.6 (2015): 512-519.

18. WC Ripka., et al. "Protein b-turn Mimetics II: Design, Synthesis, and Evaluation in the Cyclic Peptide Gramicidin S". Tetrahedron 49.17 (1993): 3609-3628. 
19. Inbal Lapidot., et al. "1,4-Dihydropyridine Cationic Peptidomimetics with Antibacterial Activity". International Journal of Peptide Research and Therapeutics 21.3 (2015): 243-247.

20. C Savio Chan., et al. "Rejuvenation' protects neurons in mouse models of Parkinson's disease". Nature 447 (2007):10811086.

21. Shatzmiller S., et al. "Blood Brain Barrier Crossing for Therapeutic and Diagnostic Agents". SM Journal of Neurological Disorders and Stroke 2.2 (2016): 1012.

22. Inbal Lapidot., et al. "a Aminoisobutyric Acid Leads a Fluorescent syn-bimane LASER Probe Across the Blood-brain Barrier". Medicinal Chemistry 12.1 (2016): 48-53.

23. Marina Kovaliov., et al. "Why Gram-Positive Bacteria are Easier to Eradicate with the N-CH3Analogs?". BAOJ Neurol 3 (2017): 046 .

24. Margaret McFall-Ngai., et al. " Animals in a bacterial world, a new imperative for the life sciences". Proceedings of the $\mathrm{Na}$ tional Academy of Sciences of the United States of America 110.9 (2013): 3229-3236.

25. https://www.sciencemag.org/news/2018/11/do-gut-bacteria-make-second-home-our-brains

26. Clara Seira Oriach., et al. "Food for thought: The role of nutritionin the microbiota-gutebrain axi". Clinical Nutritio Experimental 6 (2016): 25-38.

27. https://endpoints.elysiumhealth.com/microbiome-explainer-e345658db2c

28. Shen Liang., et al. "Alzheimer's Disease Histological and Behavioral Manifestations in Transgenic Mice Correlate with Specific Gut Microbiome State". Journal of Alzheimer's Disease 56.1 (2017): 385-390

29. Arun T Nair., et al. "Gut Microbiota Dysfunction as Reliable Non-invasive Early Diagnostic Biomarkers in the Pathophysiology of Parkinson's Disease: A Critical Review". Journal of Neurogastroenterology and Motility 24.1 (2018): 30-42.

30. Choi HH and Cho YS. "Fecal Microbiota Transplantation: Current Applications, Effectiveness, and Future Perspectives". Clinical Endoscopy 49.3 (2016): 257-265.

\section{Volume 2 Issue 8 August 2019}

(C) All rights are reserved by Shimon Shatzmiller., et al. 